\title{
Branched-Chain Fatty Acid Content Modulates Structure, Fluidity and Phase in Model Microbial Cell Membranes
}

\author{
Barmak Mostofian', Tony Zhuang², Xiaolin Cheng3,*, Jonathan D. Nickels4,*. \\ ${ }^{1}$ Department of Biomedical Engineering, School of Medicine, Oregon Health \& Science University, \\ Portland, OR 97239, USA; ${ }^{2}$ College of Medicine, University of Tennessee, Memphis, TN 38163, USA; 3 \\ Division of Medicinal Chemistry and Pharmacognosy, College of Pharmacy, The Ohio State University, \\ Columbus, OH 43210, USA; \\ 4 Department of Chemical and Environmental Engineering, University of Cincinnati, Cincinnati, $\mathrm{OH} 45221$, \\ USA.
}

This document contains:

Supporting Tables S1-S4, Supporting Figure S1-S3. 
Table S1. Head group (HG) composition of membrane systems sorted by the $n 16: 0$ mole fraction. The HG structures are visualized in Figure 1A of the main text; FA corresponds to free fatty acids that actually lack a head group structure. The total number of HGs is 420 per leaflet.

\begin{tabular}{c|ccccc}
\hline \% n16:0 & \% CL & \% PE & \% DAG & \% PG & \% FA \\
\hline 7.0 & 2.9 & 55.5 & 30.2 & 9.5 & 1.0 \\
\hline 13.6 & 2.9 & 56.3 & 29.3 & 9.6 & 1.0 \\
\hline 17.5 & 2.9 & 54.9 & 30.5 & 9.9 & 1.0 \\
\hline 22.1 & 2.9 & 56.7 & 29.6 & $\mathbf{8 . 9}$ & $\mathbf{1 . 0}$ \\
\hline 24.6 & 2.9 & 53.2 & 33.1 & 8.9 & 1.0 \\
\hline 33.2 & 2.9 & 56.9 & 31.2 & 7.1 & 1.0 \\
\hline 47.3 & 2.9 & 55.5 & 29.8 & 10.0 & 1.0 \\
\hline
\end{tabular}

Table S2. Tail group (TG) composition of membrane systems sorted by $n 16: 0$ mole fraction. The TG structures are visualized in Figure 1B of the main text. The total number of TGs is 856 per leaflet.

\begin{tabular}{ccccccc}
\hline \% $i 14: 0$ & \% $i 15: 0$ & $\%$ a15:0 & $\%$ n16:0 & $\%$ i16:0 & \% $i 17: 0$ & $\% a 17: 0$ \\
\hline 4.2 & 22.4 & 33.8 & 7.0 & 11.2 & 12.3 & 9.1 \\
\hline 3.6 & 23.0 & 32.0 & 13.6 & 10.6 & 9.8 & 7.6 \\
\hline 3.4 & 20.1 & 31.3 & 17.5 & 9.9 & 9.8 & 7.4 \\
\hline 3.2 & 21.0 & 27.8 & 22.1 & 8.6 & 10.7 & 6.6 \\
\hline 2.5 & 18.3 & 28.9 & 24.6 & 8.6 & 9.1 & 7.9 \\
\hline 2.9 & 17.7 & 25.3 & 33.2 & 6.9 & 8.7 & 5.3 \\
\hline 2.0 & 13.3 & 21.3 & 47.3 & 5.7 & 6.3 & 4.1 \\
\hline
\end{tabular}


Table S3. Anomalous diffusion exponent $2 / \mathrm{d}_{\mathrm{w}}$ and diffusion coefficient D $\left[\mathrm{nm}^{2} / \mathrm{ns}^{2 / \mathrm{dw}}\right]$ obtained from fits to the MSD at long time intervals (10 ns $<=\mathrm{t}<=50 \mathrm{~ns}$ ).

\begin{tabular}{|c|c|c|}
\hline$\% n 16: 0$ & $2 / \mathrm{dW}_{\text {long }}$ & $D_{\text {long }}$ \\
\hline 7.0 & $0.83+/-2.9 \%$ & $0.012+/-6.2 \%$ \\
\hline 13.6 & $0.85+/-1.7 \%$ & $0.010+/-4.5 \%$ \\
\hline 17.5 & $0.85+/-2.4 \%$ & $0.010+/-11.7 \%$ \\
\hline 22.1 & $0.85+/-1.8 \%$ & $0.007+/-4.7 \%$ \\
\hline 24.6 & $0.71+/-1.3 \%$ & $0.010+/-5.7 \%$ \\
\hline 33.2 & $0.83+/-1.6 \%$ & $0.006+/-4.7 \%$ \\
\hline 47.3 & $0.83+/-0.9 \%$ & $0.005+/-3.0 \%$ \\
\hline
\end{tabular}

Table S4. Anomalous diffusion exponent $2 / \mathrm{d}_{\mathrm{w}}$ and diffusion coefficient D $\left[\mathrm{nm}^{2} / \mathrm{ns}^{2 / \mathrm{dw}}\right]$ obtained from fits to the MSD at short time intervals (0.01 ns $<=\mathrm{t}<=1 \mathrm{~ns})$.

\begin{tabular}{ccc}
\hline$\%$ n16:0 & $2 / \mathbf{d w}_{\text {short }}$ & $\mathbf{D}_{\text {short }}$ \\
\hline 7.0 & $0.49+/-0.6 \%$ & $0.028+/-1.9 \%$ \\
13.6 & $0.48+/-0.7 \%$ & $0.026+/-2.9 \%$ \\
17.5 & $0.48+/-1.1 \%$ & $0.026+/-3.4 \%$ \\
22.1 & $0.45+/-0.4 \%$ & $\mathbf{0 . 0 2 0}+/-2.3 \%$ \\
24.6 & $0.42+/-1.0 \%$ & $0.019+/-2.6 \%$ \\
33.2 & $0.41+/-0.5 \%$ & $\mathbf{0 . 0 1 6}+/-3.6 \%$ \\
47.3 & $0.40+/-0.5 \%$ & $0.014+/-2.4 \%$ \\
\hline
\end{tabular}


A

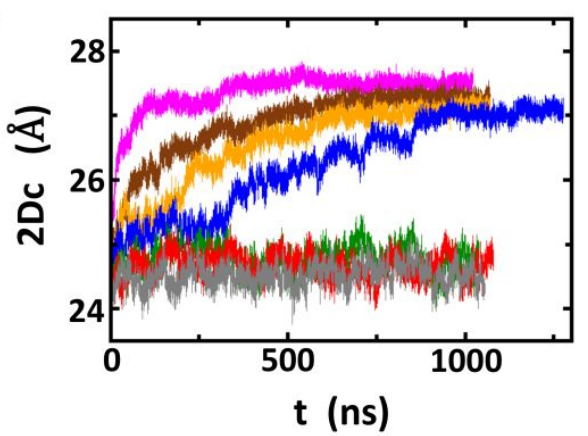

B

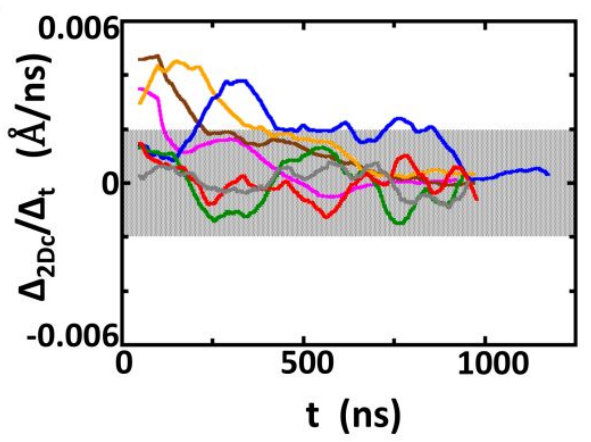

C

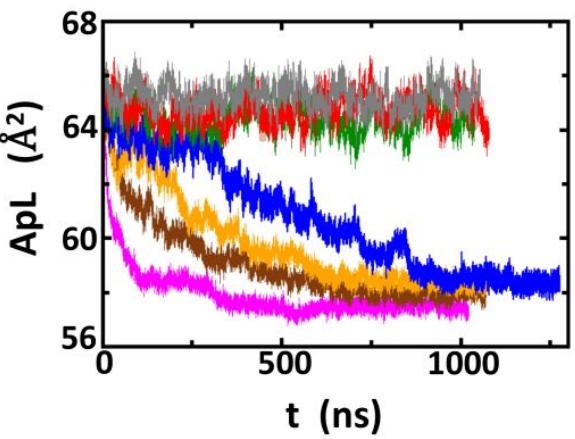

D

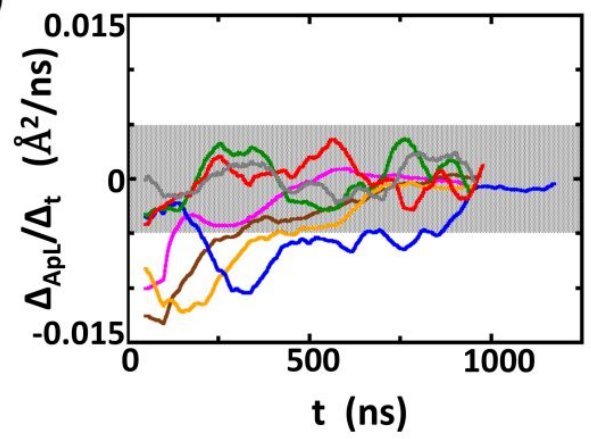

\% n16:0:

7.0

13.6

17.5

22.1

24.6

33.2

47.3

Figure S1. Bilayer equilibration. (A) The hydrophobic (or acyl) thickness, $2 \mathrm{D}_{\mathrm{c}}$, and (B) its derivative over time. (C) The area per lipid, ApL, and (D) its derivative over time. The derivatives are approximated by the difference quotient with $\Delta \mathrm{t}=100 \mathrm{~ns}$. Note that the systems with $n 16: 0>$ $20 \%$ need longer to converge than those with $n 16: 0<20 \%$. In particular, the system with $n 16: 0$ $=22.1 \%$ has been simulated for longer times to reach convergence, which is ensured for the last $250 \mathrm{~ns}$ of all simulations as indicated by the derivatives $\sim 0$. Thus, all structural properties (i.e., those shown in Figures 2, 3 and S2) are derived from the last 250 ns. 
A

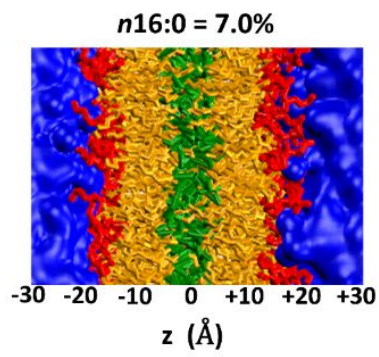

B

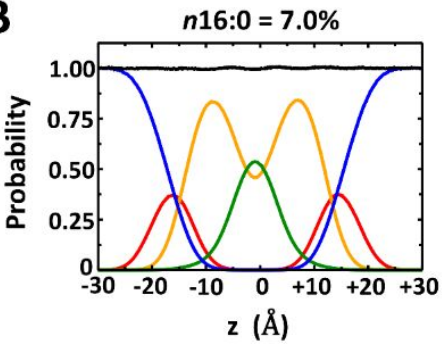

C

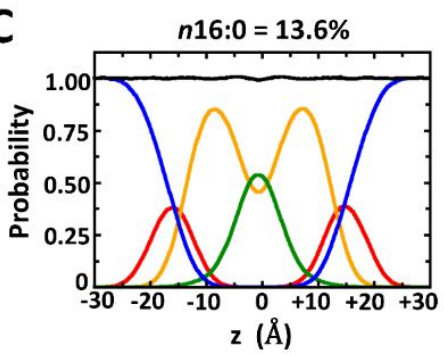

D

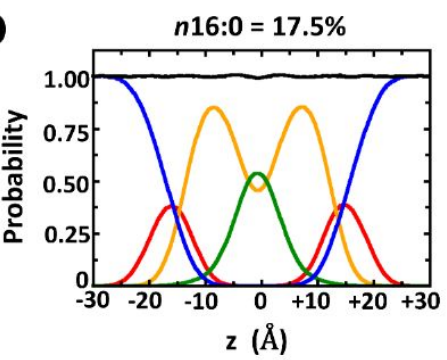

E

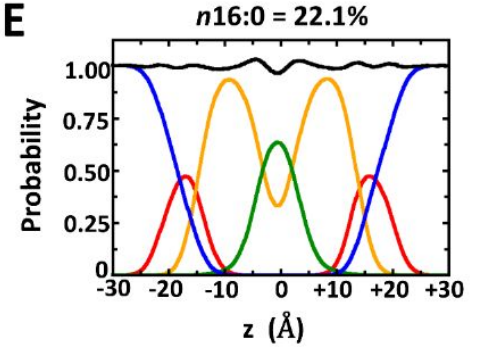

$\mathbf{F}$

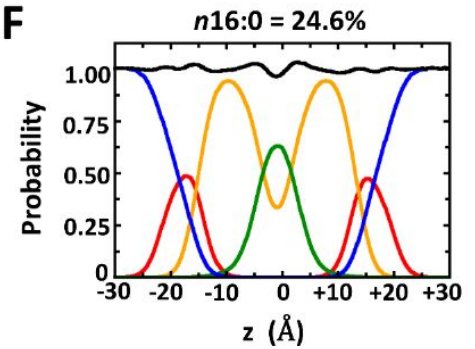

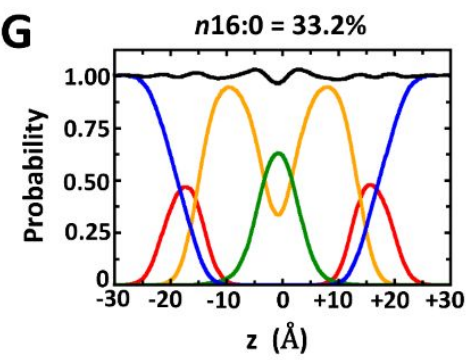

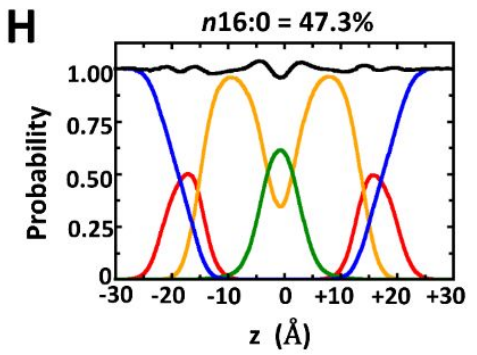

Head Groups

Tail Groups $\mathrm{CH}_{2}$

Tail Groups CH3

Water

Sum

Figure S2. Volume occupancies (or probabilities) of the various structural components of the lipid membrane along the z-axis. (A) A simulation snapshot of one bilayer with its normal (z) axis shown horizontally. The lipid head groups (carboxyl, backbone glycerol, phosphate, etc.) are shown in red, the tail group methylenes (CH2) in orange, the tail group methyls (CH3), which are essentially all branch sites, in green, and the surrounding water in blue. The superposition of occupancy profiles for each structural component from the simulated bilayer with (B) 7.0\% $n 16: 0$, (C) $13.6 \% n 16: 0$, (D) $17.5 \% n 16: 0$, (E) $22.1 \% n 16: 0$, (F) 24.6\% $n 16: 0$, (G) $33.2 \% n 16: 0$, and (H) $47.3 \% n 16: 0$, illustrating their relative positions. The colors correspond to those in (A). The sum of the component probabilities at every z-value equals 1 (shown in black). The deviation of the sum from unity around the membrane midplane $(\mathrm{z}=0)$, which becomes very pronounced for the systems with $n 16: 0>20 \%$, i.e., in $(\mathrm{E}),(\mathrm{F}),(\mathrm{G})$, and $(\mathrm{H})$, is a consequence of the greater amount of free volume around the midplane due to the reduced number of branched sites. This violates the assumption that the component volumes are independent of $\mathrm{z}$ and leads to some fluctuations in their sum, as discussed by Petrache et al. ${ }^{10}$. 

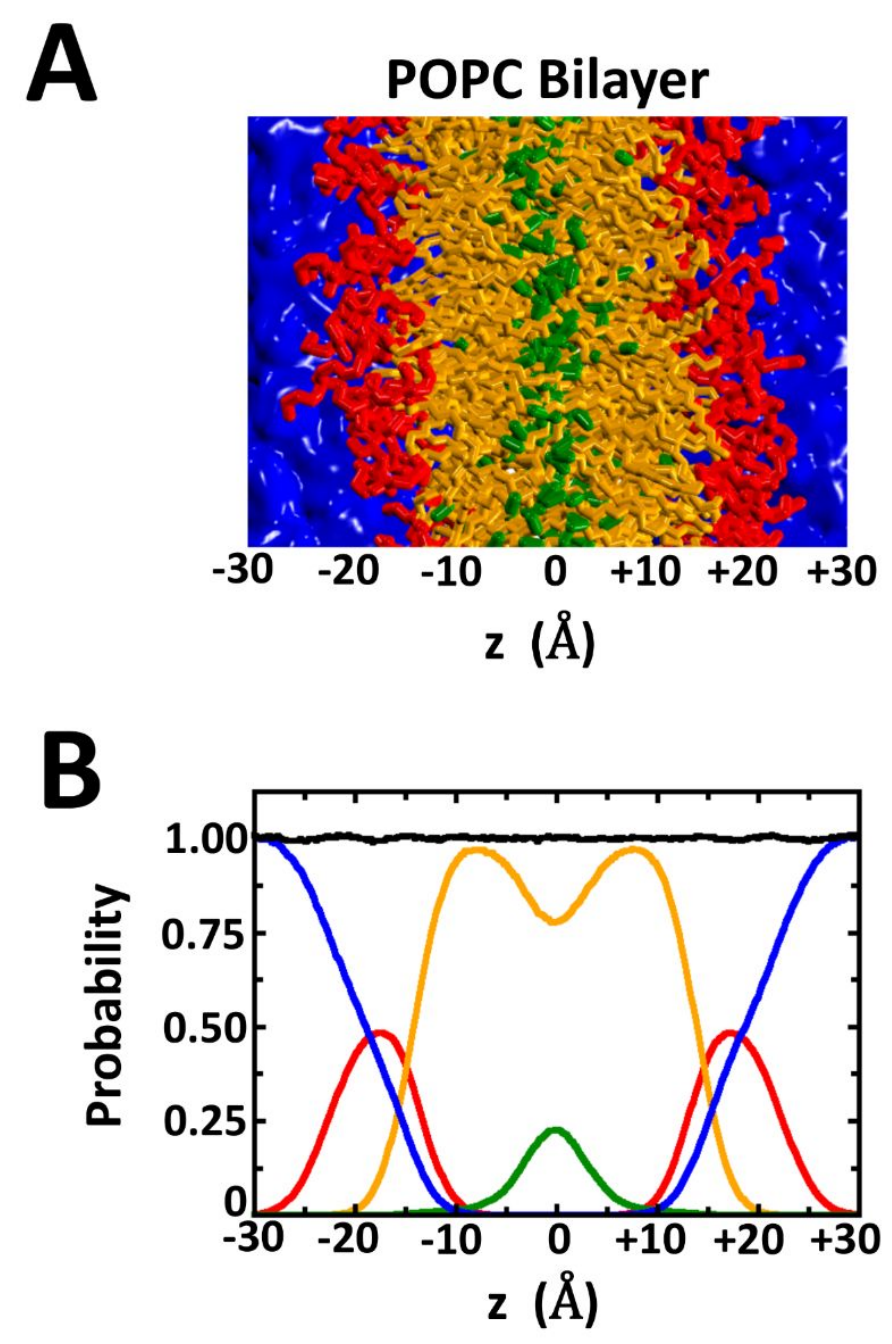

Figure S3. Volume occupancies (or probabilities) of the individual structural components of a pure-POPC lipid membrane at $300 \mathrm{~K}$ along the z-axis. (A) Snapshot of the bilayer with its normal (z) axis shown horizontally. The POPC lipid head groups are shown in red, the tail group methylenes $\left(\mathrm{CH}_{2}\right)$ in orange, the methyl groups $\left(\mathrm{CH}_{3}\right)$ in green, and water in blue. Note that in POPC lipids with two straight-chain fatty acid tails, methyl groups occur at the tail end only. This model membrane consists of 200 POPC lipids per leaflet and was simulated for $500 \mathrm{~ns}$. (B) Superposition of occupancy profiles for each structural component of POPC, illustrating their relative positions. The colors correspond to those in (A). The sum of the component probabilities at every z-value equals 1 (shown in black). The integral of leaflet profile overlap, $\mathrm{P}_{\text {overlap }}$, is $\sim 3 \%$. 